\title{
Correction to: Trocar Site Hernias in Bariatric Surgery-an Underestimated Issue: a Qualitative Systematic Review and Meta-Analysis
}

\author{
Ioannis Karampinis ${ }^{1}$ - Eliette Lion ${ }^{1} \cdot$ Maurizio Grilli $^{2} \cdot$ Svetlana Hetjens $^{3} \cdot$ Christel Weiss $^{3} \cdot$ Georgi Vassilev $^{1}$. \\ Steffen Seyfried $^{1} \cdot$ Mirko Otto $^{1,4}$ (D)
}

Published online: 21 April 2020

(C) Springer Science+Business Media, LLC, part of Springer Nature 2020

\section{Correction to: Obesity Surgery (2019) 29:1049-1057 https://doi.org/10.1007/s11695-018-03687-2}

The review was not registered for systematic reviews and meta-analyses, and should be disregarded.

The correct statement in the Abstract and in the Materials and Methods section is:

"The review was performed according to the PRISMA guidelines."

The online version of the original article can be found at https://doi.org/ $10.1007 / \mathrm{s} 11695-018-03687-2$

\footnotetext{
Mirko Otto

mirko.otto@umm.de

1 Department of Surgery, Heidelberg University, Mannheim, Germany

2 Department of Library and Information Sciences, Heidelberg University, Mannheim, Germany

3 Institute of Medical Statistic and Biomathematics, Department of Surgery, Universitätsmedizin Mannheim, Medical Faculty Mannheim, Heidelberg University, Mannheim, Germany

4 Department of Surgery, Mannheim University Medical Centre, University of Heidelberg, Theodor-Kutzer-Ufer 1-3, 68167 Mannheim, Germany
} 